\title{
Measurement of Electric Potential of a Plant using Electroconductive Cloth Sensor
}

\author{
Osamu Sakata ${ }^{\mathrm{a},{ }^{*}}$, Makoto Iwashita ${ }^{\mathrm{a}}$, Yasuyuki Satoh ${ }^{\mathrm{a}}$, Yutaka Suzuki ${ }^{\mathrm{b}}$ \\ ${ }^{a}$ Tokyo University of Science, 6-3-1 Niijuku, Katsushika, Tokyo 125-8585, Japan \\ bUniversity of Yamanashi, 4-3-11 Takeda, Kofu, Yamanashi 400-8511, Japan \\ *Corresponding Author: osakata@ee.kagu.tus.ac.jp
}

\begin{abstract}
In Japan's agriculture in recent years, "smart agriculture" has been promoted with the aim of increasing the added value of agricultural products and saving labor. As part of this effort, a number of technologies have been researched and developed for scientifically measuring the state of plants, and in this regard, we are aiming to develop technologies for continuously and accurately measuring potential fluctuations generated on the surface of plants. As of now, typical tools and methods for measuring electrical phenomena of plants have not been established, and researchers make measurements by applying electrodes, systems, and the like that are used individually for the human body. However, since these devices are used for the human body, they are often not capable of reliably and accurately measuring plants because they are loaded or unusable. Therefore, in this study, in order to measure the potential fluctuation of the leaf surface, an improved electrode which does not apply the stress to the plant as much as possible was proposed, and its effectiveness was investigated.
\end{abstract}

Keywords: electric potential, plant, smart agriculture, electroconductive cloth

\section{Introduction}

Plants provide a variety of benefits to humans. For example, it is used for absorption of carbon dioxide, food, energy source, and medicine. Further, it has an air purifying function and a healing function. It has also been reported that placing plants in an office environment improves labor efficiency. Plants have the ability to sense environmental factors, temperature, humidity, illuminance, etc. around them, and they carry out life activities while adapting to various environmental changes. In order to clarify the process of adapting to the environmental change of the plant, this study focuses on the plant biopotential as a way of observing the activity state in the plant. The plant biopotential is an electric signal generated when a plant performs some kind of information processing activity, and the potential change is generated by the influence of the plant's life activity and environmental change ${ }^{(1-4)}$. If the relationship between the environmental change and the biopotential can be clarified and environmental information can be extracted, the plant can be used as a sensor.

Studies of biopotential have revealed that biopotential is affected by temperature, water content in soil, and air volume. Variations in biopotential of a plant have also been observed for human behavior around the plant, such as human contact with the foliar surface, human access to the room being measured, etc. However, in order to clarify or evaluate the potential variation process and mechanism of plants in practice, it is still essential to examine the relationship between the physiological phenomena of plants and the biopotential reaction, and it is a major problem that the examination of this part is still inadequate. As a major factor, a tool for measuring an electric phenomenon of a plant has not been established at present, and many researchers measure electrical potential of a plant with electrodes and equipment for human body measurement. However, since these devices are used for the human body, the plants are loaded, the usability is poor, and there is a great room for improvement and contrivance. Previous studies ${ }^{(5-8)}$ have adopted in common a certain method for measuring plant biopotentials. It is a method in which two metal electrodes are directly attached to the surface of a plant, and the potential between them or between a leaf and soil is amplified by an amplifier and recorded as it is, which is 


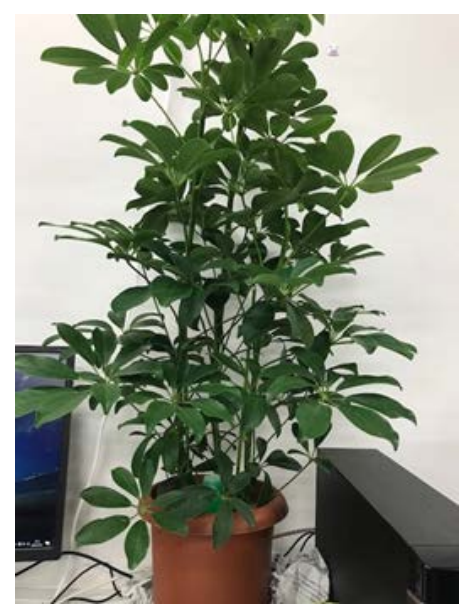

Fig. 1. Schefflera.

similar to human electromyography measurement.

With the future possibility of industrial application of the electric phenomenon measurement of plants in mind, we are working on the development of the technology for measuring the surface potential signal of a plant in a simple and reliable manner without damaging the plant. An experiment was conducted in which the electrode was modified so as not to impose any stress on the plant as much as possible. In this study, we have investigated the effect of the electrode using capacitive coupling.

\section{Measurement Method}

\subsection{Foliar Electric Potential of a Plant}

It is well known that an animal such as a human generates electric potentials from various organs and tissues constituting the body, plants also generate an electric potential, and signals in a frequency band close to that of the animal are detected. However, since the history of bioelectric research in plants is relatively new, and the accurate frequency band has not been elucidated, the signal at $100 \mathrm{~Hz}$ or less was observed in this study. In recent years, as a result of progress in a technique of measuring a microbiopotential at a $\mu \mathrm{V}$ level by an amplifier, it has been found that there is a potential difference between arbitrary two points of a plant foliage potential, and a potential variation of a cell population in contact with an electrode for potential measurement has been observed. This is called foliar potential among biopotentials. Since the foliar potential has a large area of the foliar surface in contact with the outside air, the foliar potential contains abundant biological information and exhibits an intrinsic response according to the intensity.

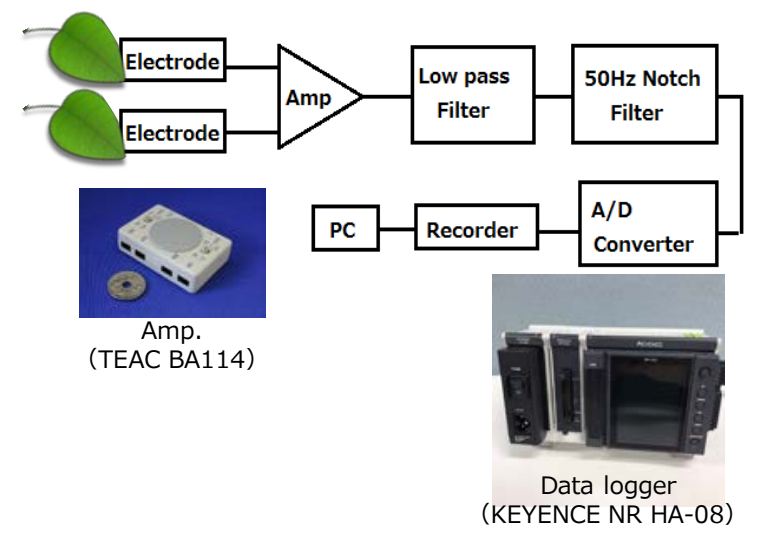

Fig. 2. Measurement System.

Plants are known to change the potential of various parts in response to external stimuli such as motion, contact, approach, vibration, sound, water, the activity of the plant in response to the temperature, humidity, and the amount of water in the air, or photosynthetic, which is the activity of the plant itself.

In an attempt to measure foliar potential, which is often performed, a way of directly contacting a plant with a metal electrode such as a human brain wave or a myoelectric potential measurement is used. The purpose of this study is to confirm that the foliar potential can be measured by changing this point.

Chefleracapoc was adopted as the experimental plant in this study (Fig. 1). The growth temperature range of cheflera is as wide as 3 to $30^{\circ} \mathrm{C}$. (optimum temperature range is 20 to $25^{\circ} \mathrm{C}$.), and maintenance and management are easy.

\subsection{Measurement System}

In this study, the foliar potential was measured by the measurement system as shown in Fig. 2. A general-purpose
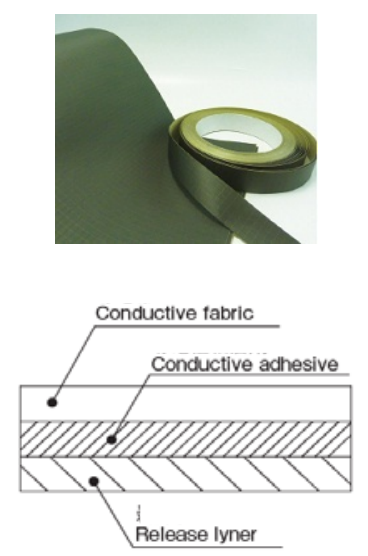

Fig. 3. Electroconductive cloth (Kitakawa Kogyo Co., Ltd.). 


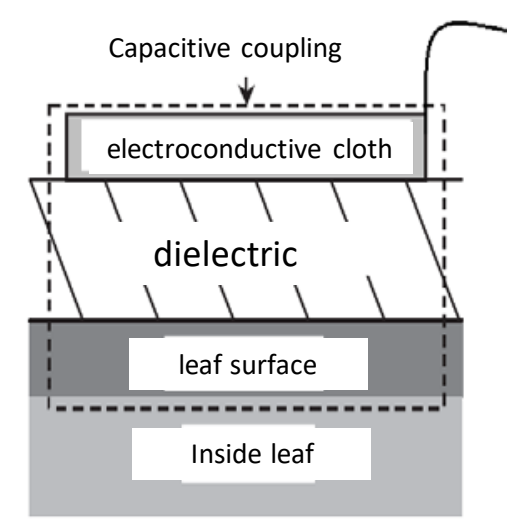

Fig. 4. Electroconductive cloth sensor.

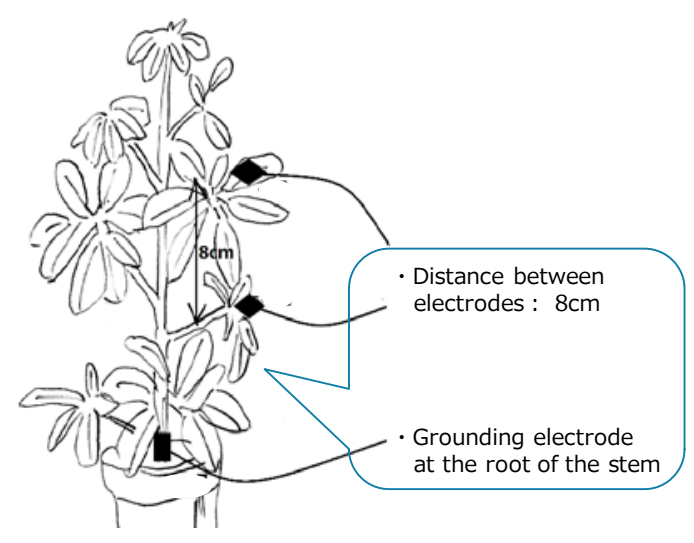

Fig. 5 Electrode arrangement.

data logger (KEYENCE NR-HA08) was used for capturing potential signals into a PC, and a biological amplifier for medical (TEAC BA1144) was used for foliar potential as a weak signal. As the electrode, a silver dish electrode for human electroencephalogram measurement and a conductive cloth (Fig. 3) were used. When conductive cloth electrodes were used based on the principle of capacitive coupling, a non-conductive felt cloth and a glass sheet were sandwiched as a dielectric between the foliage and the cloth electrodes (Fig. 4).

\section{Experimental Methods and Results}

\subsection{Comparison with conventional bioelectrodes}

In the experiment, the biopotential and the temperature in the atmosphere $\left({ }^{\circ} \mathrm{C}\right.$.) were measured simultaneously. The sampling interval was $100 \mathrm{~ms}$, the number of samples was 1801 , and the temperature at the time of measurement was always $25^{\circ} \mathrm{C}$. Figure 5 shows an outline of a sensor system

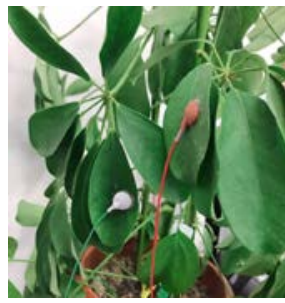

Silver electrode

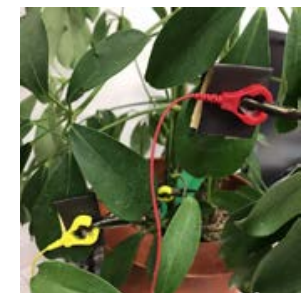

Electroconductive cloth
Fig. 6 Metal electrode (left) and electroconductive cloth electrode (right).

attached to a test plant (Chefleracapoc) used in the experiment. Electrodes were attached to two different leaf surfaces on the same stem of the test plant and kept constant at a distance of $8 \mathrm{~cm}$. The upper electrode of the leaf surface was the positive electrode, the lower electrode was the negative electrode, and the root portion of the stem closest to the soil of the test plant pot was grounded.

The potential difference obtained from the electrodes was amplified 10,000 times by an TEAC BA1144 and taken into a computer via filters, $\mathrm{A} / \mathrm{D}$ converters, and a recording device. A 3V battery was used as the power supply for the amplifiers, and the recording device was allowed to stand by for 15 minutes after starting up for warm-up, and the results of measurements after zero-point adjustments were performed were used.

First, as shown in Fig. 6, an electroencephalogram measurement plate electrode coated with a conventionally used electroencephalogram measurement paste was attached to the surface of the leaf to perform measurement. Next, measurement was also performed when leaves were sandwiched by the conductive cloth $\left(3 \mathrm{~cm} \times 5 \mathrm{~cm}=15 \mathrm{~cm}^{2}\right)$.

The following is a comparison of the results of
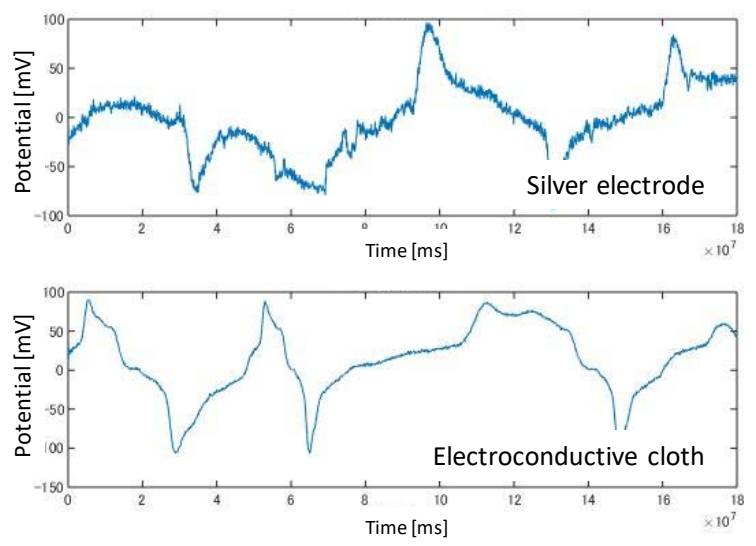

Fig. 7 Experimental results for dish electrodes (top) and conductive cloth electrodes (bottom) 


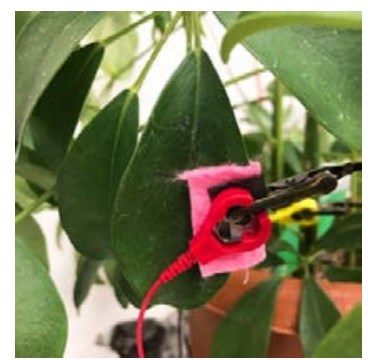

Fig. 8 A dielectric inserted between the test plant and the conductive cloth electrode.

measures on the plate electrodes and the results of measurements on the conductive cloth when attached to the foliar surface.

Fig. 7 shows that the potential difference changes by about $\pm 200 \mathrm{mV}$ in 3 minutes. Further, since the conductive cloth electrode was more firmly attached to the uneven surface of the test plant in a wider range than the measurement with the dish electrode, the noise was reduced.

\subsection{Measurement by the Capacitive Coupling Electrode}

In order to measure the biopotential by the capacitive coupling, a dielectric was inserted between the test plant and the conductive cloth electrode as shown in Fig. 8 described above. First, a commercially available cloth (material: rayon polyester, electrode portion: $4 \mathrm{~cm} \times 6 \mathrm{~cm}=24 \mathrm{~cm}^{2}$, thickness of one sheet: $0.9 \mathrm{~mm}$, thickness of two sheets: 1.7 $\mathrm{mm}$, thickness of three sheets: $2.5 \mathrm{~mm}$ ) was sandwiched as a dielectric, and measurement was performed with a sampling interval of $100 \mathrm{~ms}$

Additionally, in order to examine the application range of the prototype device, two or three pieces of a commercially available cloth were superposed on each other, and the change in the biopotential was recorded. The result is shown in Fig. 9. The graph shows the conductive electrodes of 0 cloth, 1 cloth, 2 cloth, and 3 cloth in this order from the top.

Focusing on the result of superimposing the fabrics in Fig. 9, at the output of superimposing one sheet, a potential difference slightly larger than that at the time of not interposing the fabrics is generated, and the waveforms have the same shape. Further, in the case where two sheets are superimposed, an output of the same level as that in the case where one sheet is superimposed is obtained, and the waveform also has many low-frequency components. It can be seen that in the case where the last three sheets are superimposed, the amplitude is considerably large and the
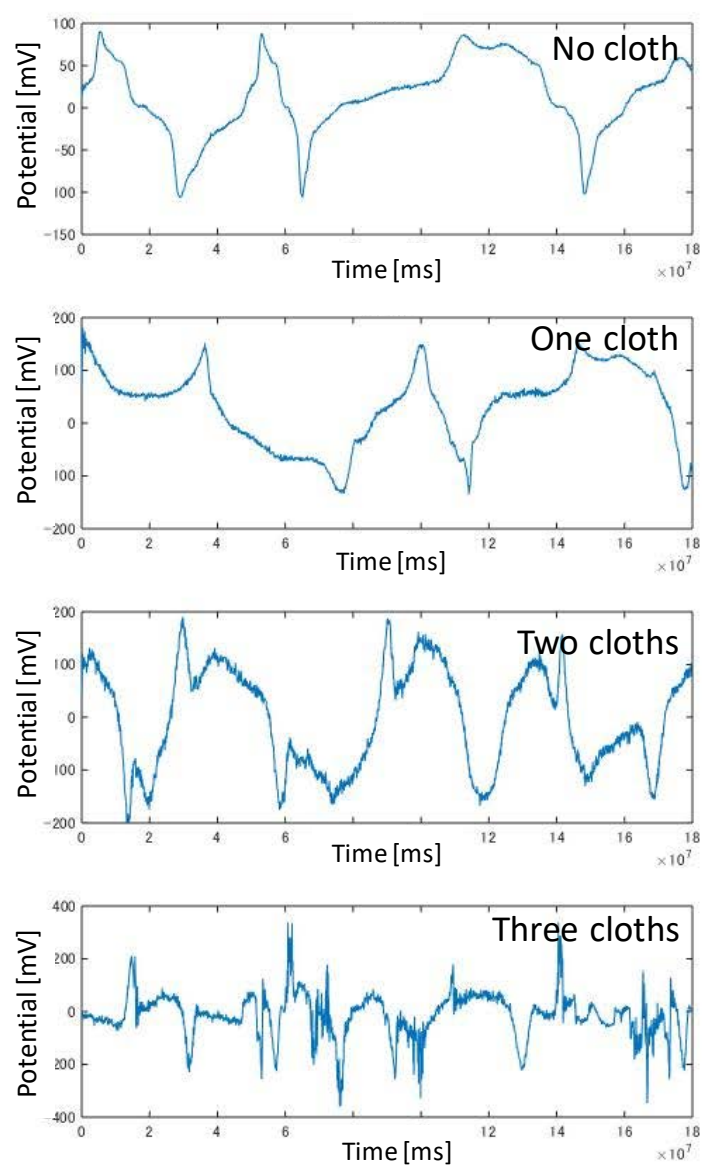

Fig. 9 Output waveform when potential was measured with overlapping the dielectric fabrics.

noise is also large, and the biopotential waveform is almost completely disappeared. When this result is converted into the thickness of the cloth, it is understood that about $0.1 \mathrm{~mm}$ to $2.0 \mathrm{~mm}$ is suitable for the measurement by the capacitive property.

Next, measurement was performed by changing the dielectric. A commercially available glass sheet (electrode part: 1 sheet thickness: $1.0 \mathrm{~mm}, 2$ sheets thickness: $2.0 \mathrm{~mm}$, 3 sheets thickness: $3.0 \mathrm{~mm}$ ) was sandwiched between the test plant and the conductive cloth electrode. The sampling interval was $100 \mathrm{~ms}$, and 1 , 2, and 3 samples were used in an overlapping manner, and changes in the biopotential were recorded. The results are shown in Fig. 10. In the graph, 0 sheets of glass, one sheet of glass, two sheets of glass, and three sheets of glass are arranged in this order from the top.

Focusing on this result, it was found that a potential difference slightly larger than that in the case where glass is 

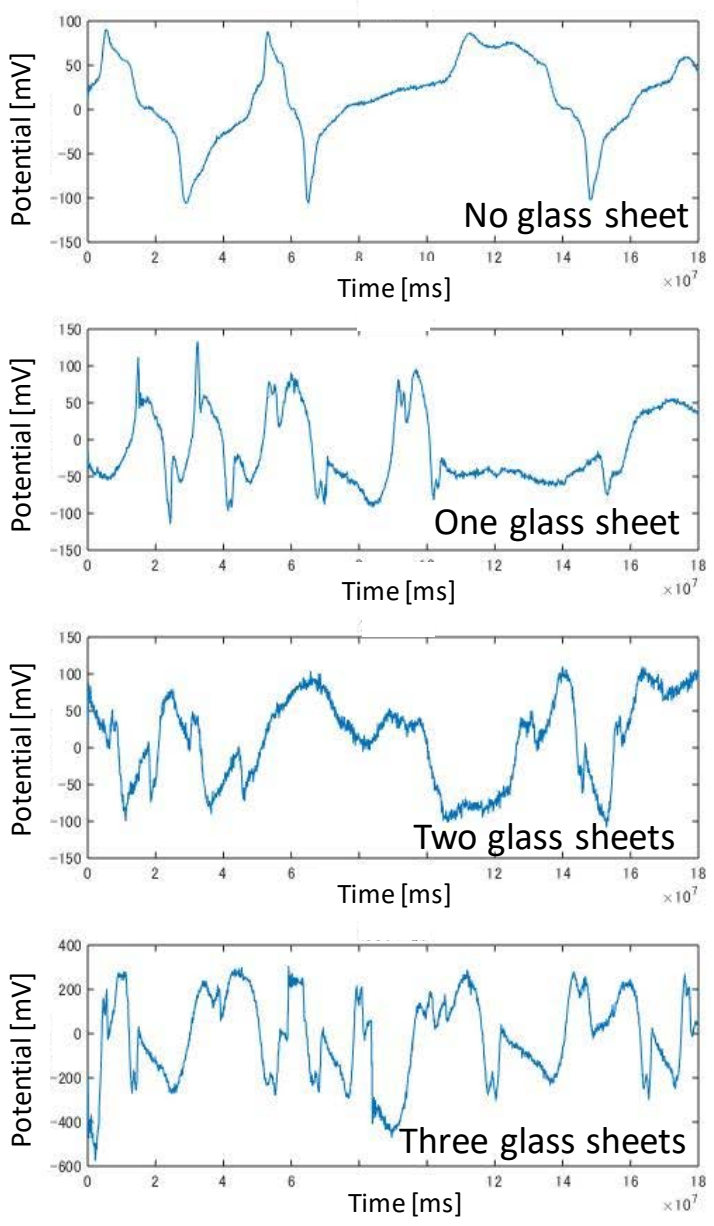

Fig. 10 Output waveform when potential was measured with overlapping the glass sheets.

not interposed is generated in the output in which one sheet is superposed, and the waveform also fluctuates greatly at intervals of about 10s. It is considered that this is because the glass is heavy and the plant is loaded. In the case where two sheets were superposed, an output of the same level as that obtained when one sheet was superposed was obtained, and the waveform was similar to that obtained when one sheet was superposed. When the last three sheets are stacked, the amplitude is considerably large in the case of the glass as in the case of the cloth, and it is understood that the noise is also large.

The relative dielectric constant of the glass is 4.5 to 5.2 , which is larger than that of a commercially available cloth, and therefore, the glass tends to have a capacitance. The theoretical shows that the voltage (potential difference) becomes smaller because the charge stored in the capacitor is more constant in the case of sandwiching the glass than in the case of the equation. Therefore, the thickness can be measured more than when the dielectric material is a cloth. However, since the weight load on the test plant is large in the case of using glass this time, it is considered that accurate waveforms are only required until two sheets are sandwiched. When this result is converted into the thickness of the glass, it is understood that about $0.1 \mathrm{~mm}$ to $1.5 \mathrm{~mm}$ is suitable for the measurement by the capacitive property. However, since there is a slight impact of air existing between the cloth and the cloth and between the glass and the glass in either case of the cloth and the glass, the limit value at the time of insertion is expected to be slightly larger than the abovementioned value.

\subsection{Long-time Measurement}

In the case of the measurement method in which the plate electrode is directly attached to the leaf surface, the deterioration or sore of the surface of the test plant occurs by attaching the conductive glue for a long time, and therefore, the measurement is difficult for a long time. Therefore, in this study, the dielectric cloth was sandwiched between the conductive cloth electrode and the leaf surface, and the measurement was carried out for 2 hours.

The materials of the dielectric cloth and the electrodes of the conductive cloth were the same as those of the above mentioned experiments. Sampling was performed at a sampling interval of 0.1 second, and the temperature at the time of measurement was always set to $25^{\circ} \mathrm{C}$.

Figure 11 shows the results obtained when two cloths were passed therethrough. Figure 11 shows the results after (a) 0 minutes, (b) 30 minutes, (c) 60 minutes, and (d) 120 minutes from the start of the experiment.

In addition, since it was judged that the measurement with the glass sheet as a dielectrode could not be performed for a long time due to the weight of the electrode portion, only the measurement with rayon polyester cloth was performed for a long time.

From this result, firstly, when the entire waveform was compared, a large change in the waveform such as sandwiching three cloths could not be obtained, and therefore, the deterioration with the passage of time was hardly confirmed. Therefore, it is basically considered that the device is stable and durable for a long time.

When the signal is stable in this manner, analysis of the measurement signal becomes easy, and there is a possibility that biopotential information from the potential difference signal can be further extracted. 


\section{Conclusion}

The biopotential derivation of the test plant by the conductive electrode through two kinds of dielectric materials (rayon polyester cloth and glass sheet) was realized by the combination of the application of capacitive coupling and high-performance amplifier for anthropometry. As a result, it was also confirmed that the obtained biopotential contained waveform information similar to that of the dish electrode which was conventionally measured. With respect to the thickness of the fabric as the dielectric, it was confirmed that a stable measurement result was obtained if the fabric was $2.0 \mathrm{~mm}$ and the glass was $1.5 \mathrm{~mm}$, and that the stability of the device accompanied by the long-time
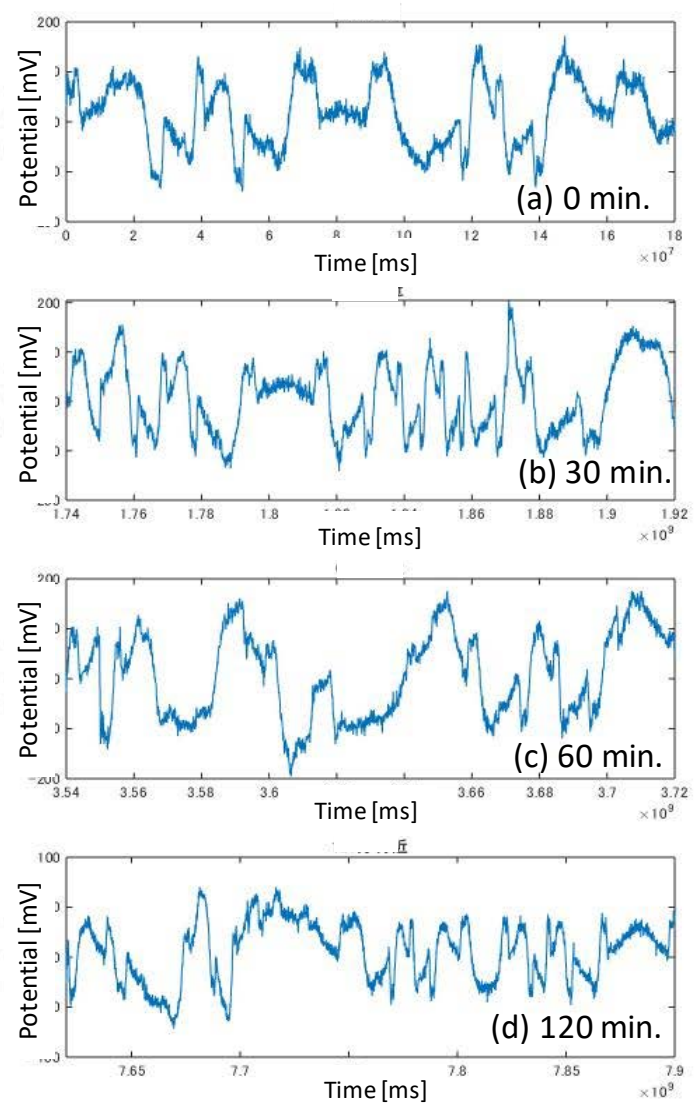

Fig. 11 Long-term measurement when two dielectric materials (cloth) are sandwiched. The results after (a) 0 minutes, (b) 30 minutes, (c) 60 minutes, and (d) 120 minutes from the start of the measurement. measurement was hardly changed. From these results, it can be said that the measurement method and the circuit configuration proposed in this study are effective for measuring the potential from the electrode through the conductive cloth.

\section{References}

(1) Liu Kai, Xi Gang, Fan Lin-lin, Meng Xiao-li, Gao Xiang, Liu Qing, "The Changes of Electrical Signals in Corn at Different Temperatures”, Procedia Environmental Sciences 10, pp.39-44, 2011.

(2) Xiaofei Yan, Zhongyi Wang , Lan Huang, Cheng Wang, Ruifeng Hou ,Zhilong Xu , Xiaojun Qiao, "Research progress on electrical signals in higher plants", Progress in Natural Science 19, pp.531-541, 2009.

(3) Paz Merelo, Esther Botterweg Paredes, Marcus G Heisler, Stephan Wenkel, "The shady side of leaf development: the role of the REVOLUTA/KANADI1 module in leaf patterning and auxin-mediated growth promotion”, Current Opinion in Plant Biology, 35, pp.111-116, 2017.

(4) Federico Brilli, Silvano Fares, Andrea Ghirardo, Pieter de Visser, Vicent Calatayud,Amalia Muñoz, Isabella Annesi-Maesano, Federico Sebastiani, Alessandro Alivernini, Vincenzo Varriale, and Flavio Menghini, "Plants for Sustainable Improvement of Indoor Air Quality”, Trends in Plant Science, Vol. 23, No. 6, pp.507-512, 2018.

(5) S. Hirobayashi, Y. Tamura, T.Yamabuchi, and T. Oyabu, "Mnitoring of Human Activity using Plant Bioelectric Potential,” IEJ Trans. SM, Vol.127-E, No.4, pp.258-279, 2007. (in Japanese)

(6) J. Fromm and S. Lauther, "Electrical signals and their physiological significance in plants", Plant, Cell and Environment, Vol.30, pp.249-257, 2007.

(7) Y. Hasegawa, S. Asada, T. Katsube, and T. Ikeguchi, "Analysis of Bioelectrical Potential When Plant Purifies Air Pollutoin”, IEICE Trans. Electron., Vol.E87-C, No.12, pp.2093-2098, 2004.

(8) L.A. Gurovich and P. Hemosilla, "Electric signaling in fruit trees in response to water application and lightdarkness conditions", J. Plant Physiology, Vol.166, pp.290-300, 2009. 